\title{
Heidelberg 1938
}

Die 52. Heidelberger Tagung stand im Zeichen der Zuerken-nung der Grae/e-Medaille. Es gait zum 6. Male den Mann auszu-zeichnen, der laut Statut sich innerhalb des Zeitraumes der letzten zehn Jahre unter den Zeitgenossen, ohne Unterschied der Natio-nalität, die größten Verdienste urn die Förderung der Augenheil-kunde erworben hatte. Es mag vielleicht nicht unangebracht sein, sich bei dieser Gelegenheit der Manner zu erinnern, denen bisher in Heidelberg diese größte Ehrung, die die deutsche Ophthalmologie zu vergeben hat, zuteil wurde.

Die Medaille wurde erstmals 1886 Hermann Helmholtz ver-liehen, dem die Ophthalmologie den Augenspiegel zu verdanken hat. Zehn Jahre später war es der Heidelberger Kliniker Theodor Leber, der vor allem die von A. v. Graefe gewonnenen klinischen Erkenntnisse auf eine breite pathologisch-anatomische und na-mentlich auch physiologische Basis gestellt hatte. 1906 folgt Ewald Hering, der Leipziger Sinnesphysiologe, der durch seine Arbeiten über die Farbenempfindung, den Raumsinn und den Be-wegungsapparat des Auges der Ophthalmologie neue Wege er-schlossen hatte. Nach längerer Zwischenzeit wurde das Ehren-zeichen erst 1922 wieder vergeben. Es gait dem Münchener Kliniker Carl von Heß, der sich besonders um die Erkenntnis der vergleichenden Physiologie des Sehens Verdienste erworben und die Heríngschen Lehren nutzbringend in der Ophthalmologie an-gewendet hatte. 1928, zur Feier des 100. Geburtstages v. Graefes, wurde die Medaille dem Schweden Allvar Gullstrand verliehen in Anerkennung für die bahnbrechenden Leistungen auf dem Ge-biete der Optik, der Brillenlehre und vor allem auch für die Er-findung der Spaltlampe.

Die diesjährige Tagung hat den Schweizer Jules Gonin mit der Grae/e-Medaille ausgezeichnet. Es ist das erstemal, daß die Auszeichnung einem Toten verliehen worden ist. Aber zweifel-los ist Gonins Erkenntnis, daß die Netzhautablösung fast stets durch eine Loch- oder Rißbildung eingeleitet wird und daß nur der Verschluß dieses Risses Heilung des Leidens ermöglicht, in Heidelberg 1938

335

therapeutischer Hinsicht das Wertvollste, was das letzte Dezen-nium der Ophthalmologie geschenkt hat. Daß einem Nicht-Deutschen heute, in den Zeiten nationalen Abschlusses, die Ehrung zuteil wurde, stellt ein tröstliches Zeichen dafür dar, daß die Er-kenntnis, Wissenschaft sei nicht an politische Grenzen gebunden, noch nicht verloren gegangen ist.

Das Programm der diesjährigen wissenschaftlichen Sit-zungen war außerordentlich vielseitig und reichhaltig. Ueber-blicken wir die Gesamtheit des Gebotenen, so bleiben einzelne Mitteilungen als besonders eindrücklich und fruchtbringend haften. So bringen Seídels Untersuchungen über die Akkom-modation ini albinotischen Menschenauge einen wertvollen Be-weis fur die Richtigkeit der Helmholtzschen Akkommodations-lehre. Die Frage nach dem Wesen der Schielamblyopie hat durch Harms weitgehende Klärung erfahren. Praktisch wertvoll schei-nen besonders auch die Untersuchungen von Amsler über die sogenannten «formes frustes» des Keratokonus zu sein, Untersuchungen, die eine wesentliche Erweiterung des Keratokonus-begriffes zur Folge haben dürften. Ein großer Teil der Tagung war dem Thema der normalen und kranken Linse gewidmet. Unter den gebotenen Referaten war es hauptsächlich der 
Vortrag von H. K. Mailer, der uns in das für die Entstehung des Alters-stares so wichtige Geschehen des normalen und krankhaften Linsenstoffwechsels Einblick gewährte und auch neue therapeu-tische Möglichkeiten erschloß. Für den pathologisch-anatomisch Interessierten waren die Mitteilungen von Reichling über die sogenannten Grenzzonen des Auges von Bedeutung. Ein großer Teil der Vorträge befaßte sich mit Verbesserungen unserer diagnosti-schen Methoden. Eine Bereicherung bringen in dieser Hinsicht namentlich das neue Dynamometer und der Lidkraftmesser von H. K. Müller; auch das neue Orbitographieverfahren von Thíel dürfte sich als nutzbringend erweisen. Sehr praktisch ist die neue Lichtsinnprüfmethode nach Comberg. Den operativ interessierten Ophthalmologen mußte Löhleins Bericht über seine Resultate bei Keratoplastik interessieren und zur Nachahmung anregen. Der Vortrag von Fleischer, der sich mit der Frage befaßte, ob die Be-handlungsmöglichkeit eines Erbleidens die Anwendung des Gesetzes zur Verhütung erbkranken Nachwuchses ausschließt, zeigte namentlich in der anschließenden Diskussion die großen Diver-genzen, die unter den einzelnen Ophthalmologen in der Auffassung

336

Deutsche Ophthalmologische Gesellschaft

solcher Fragen bestehen. Behe zzigenswert erscheint die von Bucklers unterstrichene Tatsache, daß in der neuen Auflage des Gesetzes ausdrücklich bestimmt ist, daß Unfruchtbarmachung nur bei komplizierten Staren in Frage kommt, bei denen auch die operative Behandlung keine befriedigenden Erfolgsaussichten bietet, wobei man unter «kompliziert» jene Linsentrübungen ver-steht, die gleichzeitig mit anderen körperlichen oder geistigen Minderwertigkeiten einhergehen.

Wir weichen in der Folge von der üblichen Art der Gesell-schaftsberichterstattung ab und geben die Vorträge nicht in chronologischer Reihenfolge, sondern ihrem Inhalt entsprechend geordnet wieder, wobei wir uns auf die Zusammenfassung des Wesentlichen beschränken.

F. Rintelen.

Deutsche Ophthalmologische Gesellschaft.

Berícht liber die 52. Tagung in Heidelberg vom i.-6. Juli 1938. Von F. RINTELEN, Basel. 1. Lider und Tränenorgane.

A. Fuchs stellt die Frage auf, ob es sich entsprechend der Theorie Schalls beim Chalazion wirklich um eine Lipogranulomatose handelt. Dagegen spricht die Tatsache, daß sich das Chalazion durchaus nicht imnier in der Umgebung der tarsalen Talgdrüsen entwickelt. Fuchs konnte seine Entwick-lung in frischen Fallen vielfach vo $\tau$ dem Tarsus, im Bereich der Zilien und im freien Lidrande feststellen. Histologisch findet sich in den ersten Stadien des Chalazions ein Granulationsgewebe, das reich ist an eosinophilen Zellen, Lymphozyten und Plasmazellen. Die Genese des Chalazions ist nach Fuchs noch nicht geklärt. - In der Aussprache weist Pillat darauf hin, daß es sich beim Chalazion eigentlich um einen klinischen Begriff handelt, hinter dem sich histologisch und ätiologisch verschiedene Krankheiten verbergen können. So tritt z. B. bei echtem Vitamin-A-Mangel die häufig multipel und in kurzer Zeit einsetzende Chalazionbildung im Anschlusse an den hyperkeratotischen Verschluß de_s Ausführungsganges der Me ¿fromschen Drüsen auf.

2. Konjunktiva.

G. Grüter hat beim Frühjahrskatarrh die Mikrostruktur entzündeter Bindehaut- und Hornhautepithelien untersucht und festgestellt

daß morpho-logisch betrachtet die entzündlichen Veränderungen in der Zelle im Sinne einer Abwehrreaktion zu deuten sind 
wie sie sich nach den gleichen Prin-zipien auch beim Trachom abspielen. Irgendwelche zellfremde Mikrobefunde konnten nicht erhoben werden. 